\title{
Dialectical Behavior Therapy for the Treatment of Borderline Personality Disorder
}

\author{
Michael A. Mancini and Shannon Cooper-Sadlo
}

\section{LEARNING OBJECTIVES}

By the end of this chapter, you should be able to:

1. develop an understanding of the prevalence, pathogenesis, symptomology, and course for borderline personality disorder (BPD);

2. identify the biological, psychological, and social factors that contribute to the development of BPD;

3. identify screening and assessment practices for the effective diagnosis of BPD;

4. understand the historical development, central theoretical constructs, structures, processes, evidence base, and limitations of dialectical behavior therapy (DBT); and

5. identify and effectively apply the specific practices and interventions of DBT.

\section{INTRODUCTION}

Borderline personality disorder (BPD) causes significant mental distress and impairment in psychosocial functioning (American Psychiatric Association [APA], 2013; Gunderson et al., 2018). The condition is marked by emotional dysregulation, unstable personal relationships, impulsivity, chronic anger, and identity disturbances (APA, 2013). A person with this condition often engages long-standing patterns of maladaptive coping responses that can include substance use, self-injury, suicidal behavior, aggression, and other reckless behavior. The condition is often comorbid with other psychiatric conditions including mood and anxiety disorders, eating disorders, substance abuse, posttraumatic stress disorder (PTSD), and other personality disorders (APA, 2013; Lenzenweger et al., 2007; Shea et al., 2004; Zanarini et al., 1998; Zimmerman \& Mattia, 1999). While prolonged impairment in psychosocial functioning and symptom relapses are common, lasting improvement in a wide range of areas can be expected over time (Gunderson et al., 2011; Skodol et al., 2005b).

Dialectical behavior therapy (DBT; Linehan, 1993, 2015) is an intensive psychotherapy based on the cognitive behavioral model that uses individual and group sessions to help people with BPD develop adaptive coping strategies to manage symptoms and improve functioning (Linehan, 1987, 1993, 2015). DBT has been widely studied and has been found to be an effective psychotherapeutic approach to treating BPD (Cristea et al., 2017; Linehan et al., 2006; Stoffers et al., 2012). 


\section{OVERVIEW AND CLINICAL FEATURES OF BORDERLINE PERSONALITY DISORDER}

There are several interrelated symptomatic clusters that comprise BPD. Sanislow et al. (2000) used factor analysis to discover three main factors that comprise BPD symptoms: (1) emotional dysregulation (e.g., anger, mood instability), (2) problems relating to other people (e.g., unstable personal relationships) and to oneself (e.g., identify disturbance and feelings of emptiness), and (3) problems managing behavior (e.g., impulsive behavior, recklessness, chronic self-harming, and suicidal behavior).

A hallmark sign of BPD is emotional dysregulation, which involves affective instability, chronic feelings of anger, and an intense fear of rejection and abandonment (Sanislow et al., 2000). This is the most common symptom found in 95\% of BPD cases (McGlashan et al., 2005). Affective instability involves drastic fluctuations from joy to irritability and anger to intense sadness over the course of a single day. Affective instability and chronic anger have also been identified as one of the most pervasive, persistent, and stable traits of BPD (McGlashan et al., 2005).

A second clinical feature of BPD is unstable personal relationships with significant others, partners, caregivers, therapists, and close family members (APA, 2013). Unstable interpersonal relationships have been found in 79\% of BPD cases (McGlashan et al., 2005). Persons with BPD often require a high degree of reassurance and support due to an intense fear of rejection and abandonment. Research indicates that persons with BPD may be inherently hypersensitive to negative emotional stimuli such as negative feedback or criticism, negative or ambiguous facial expressions, or perceived lack of optimal support from caregivers, friends, and significant others (Donegan et al., 2003; Korfine \& Hooley, 2000). They may misinterpret mild negative critiques or ambiguous responses as rejection or abandonment resulting in anger or depression, which can lead to emotional outbursts, cruelty, aggressiveness, self-harm, suicidal behaviors, substance abuse, and sabotaging of relationships or personal goals (Lieb et al., 2004).

A third feature of the disorder is an unstable self-image or identity and a chronic sense of emptiness (Gunderson et al., 2018; Lieb et al., 2004). Chronic emptiness can lead to a pervasive sense of unfulfillment causing persons with BPD to become easily bored and restless. While searching for a sense of excitement and fulfillment, persons with BPD may engage in reckless, thoughtless, or ill-advised behaviors. While persons with BPD typically view themselves in negative terms, the lack of being moored in a stable identity can lead to drastic changes in behaviors, relationships, opinions, and points of view (APA, 2013; Gunderson et al, 2018).

A fourth common feature of the disorder that can affect up to $81 \%$ of patients is a tendency toward impulsive and reckless behaviors (Lieb et al., 2004; McGlashen et al., 2005). These behaviors can include engaging in physical or verbal aggression, substance abuse, unsafe sex, reckless driving, imprudent spending of money, quitting jobs, binge eating, and abruptly ending relationships, among other self-damaging behaviors. Persons with BPD have been found to have impairment in many cognitive areas of functioning including attention, memory, planning, cognitive flexibility, and problem-solving (Ruocco, 2005). These deficits may contribute to impulsive behavior seen in persons with this condition. The combination of affective instability and impulsivity inherent in BPD can make a person with the disorder highly susceptible to maladaptive coping behaviors in the face of stressful life events. For instance, persons with BPD often tend to engage in the act of "splitting" by seeing people as either all-good or all-bad (Gunderson \& Links, 2008). Persons who provide optimal support are often idealized but can quickly be denigrated if support wanes. This response may be due to the fear of abandonment and/or a sense of chronic emptiness inherent in the disorder. This can invoke aggressive, angry, cruel, or manipulative responses. Persons with BPD may also withdraw and become depressed leading to engagement in self-harming or suicidal behavior (Lieb et al., 2004).

Lastly, persons with BPD engage in frequent suicidal behavior (e.g., suicidal ideation, planning/intent, attempts, and completion) as well as self-harming or non-suicidal self-injury 
(NSSI) such as cutting, burning, head-banging, or punching oneself (APA, 2013; Zanarini et al., 2013). Persons with BPD are at an increased risk for suicide and complete suicide at a high rate (APA, 2013; Black et al., 2004; Gunderson et al., 2018; Lieb et al., 2004; Paris, 2002; Pompili et al., 2005). Studies indicated that as high as $75 \%$ of persons with BPD attempt suicide with approximately 10\% completing suicide. Important risk factors for suicide include prior attempts, depressive symptoms, access to lethal means, alcohol and substance misuse use, hopelessness, impulsivity, and social and vocational maladaptive behaviors (APA, 2001; Black et al., 2004; Soloff \& Chiappetta, 2012).

\section{Epidemiology, Onset, and Course}

At any given point in time, the prevalence of BPD in the United States is 1.6\%, while the lifetime prevalence of the condition is approximately 6\% (Grant et al., 2008; Lenzenweger et al., 2007). In clinical settings, BPD has been identified in $20 \%$ of psychiatric inpatients and 9 to $10 \%$ of mental health treatment outpatients (APA, 2013; Zimmerman et al., 2005). Population studies have also shown no statistical differences in prevalence rates between men and women (Grant et al., 2008; Lenzenweger et al., 2007). However, in clinical settings, women comprise $75 \%$ of BPD diagnoses (APA, 2013). This suggests that women may be more likely to seek treatment than men, or it may reflect systemic biases in diagnostic practice.

Research has indicated that persons with BPD are more likely to experience alcohol and drug use disorders (Grant et al., 2015, 2016). On average, persons with BPD have over three additional psychiatric comorbid disorders with $85 \%$ having at least one comorbid disorder (Lenzenweger et al., 2007). The most common comorbid disorders include major depressive disorder (MDD) (83\%), substance use disorder (64\%), PTSD (56\%), eating disorders (53\%), and panic disorder (48\%; Shea et al., 2004; Zanarini et al., 1998; Zimmerman \& Mattia, 1999).

Like most personality disorders, a diagnosis of BPD usually occurs in late adolescence and early adulthood (APA, 2013). Despite long-held assumption about the chronic nature of the condition, longitudinal studies have shown high remission rates. In one study of 175 patients, $85 \%$ met less than three diagnostic criteria for at least 12 months at 10-year follow-up (Gunderson et al., 2011). Another longitudinal study of nearly 300 patients demonstrated 10-year remission rates (defined as not meeting diagnostic criteria for at least two years) of 91\% and 16-year rates to be as high as $99 \%$ (Zanarini et al., 2006, 2010, 2012). Remission was prolonged with almost $80 \%$ achieving remission for 8 years or more (Zanarini et al., 2012). Relapse rates in these studies ranged from 12\% (Gunderson et al., 2011) to 34\% (Zanarini et al., 2012). While improvement in many symptom domains occurred, impairment in psychosocial functioning (i.e., social relationships) was persistent over time indicating that persons with BPD experience a wide range of symptomatic improvement, yet continue to struggle with impairment in social domains (Gunderson et al., 2011; Skodol et al., 2005a).

\section{Assessment and Diagnosis}

The assessment of BPD should encompass a multidimensional approach that pays particular attention to suicidal and NSSI, violence, psychiatric symptoms, family history, substance use, childhood adverse events, and trauma. In addition to standardized instruments and client selfreport, clinicians should collect information from observations, medical records, and collateral contacts in the person's social network.

Several screening and assessment forms are available for clinical use. A common and simple screening instrument with good psychometrics is the 10-item McLean Screening Instrument for BPD (Zanarini et al., 2003). The measure uses yes and no responses to 10 items that closely track diagnostic criteria for BPD. A more in-depth diagnostic interview instrument with good psychometric properties is the Revised Diagnostic Interview for Borderlines (Zanarini et al., 2002). 
Section 2 of the Diagnostic and Statistical Manual of Mental Disorders (5th ed.; DSM-5; APA, 2013) lists the following diagnostic criteria for BPD (APA, 2013, pp. 663):

A pervasive pattern of instability of interpersonal relationships, self-image, and affects, and marked impulsivity, beginning in early adulthood and present in a variety of contexts as indicated by five (5) or more of the following:

1. Frantic efforts to avoid real or imagined abandonment (excluding suicidal behaviors covered in criterion 5)

2. A pattern of unstable and intense interpersonal relationships characterized by alternating between extremes of idealization and devaluation

3. Identity disturbance; markedly and persistently unstable self-image or sense of self

4. Impulsivity in at least two areas that are potentially self-damaging (e.g., spending, sex, substance abuse, reckless driving, binge eating). Excluding suicidal behaviors covered in criterion 5

5. Recurrent suicidal behavior, gestures, or threats, or self-mutilating behavior

6. Affective instability due to a marked reactivity of mood (e.g., intense episodic dysphoria, irritability, or anxiety usually lasting a few hours and only rarely more than a few days)

7. Chronic feelings of emptiness

8. Inappropriate, intense anger or difficulty controlling anger (e.g., frequent displays of temper, constant anger, recurrent physical fights)

9. Transient, stress-related paranoid ideation or severe dissociative symptoms

\section{Differential Diagnosis}

BPD shares many symptoms with other psychiatric disorders. The two disorders that are most similar to BPD are MDD and Bipolar Disorder I and II. MDD is often comorbid with BPD, and if a client meets full criteria for MDD and BPD, both conditions can and should be diagnosed. However, the negative mood symptoms and suicidality experienced across both conditions can make differential diagnosis difficult. When differentiating, it should be noted that the negative mood symptoms experienced during a major depressive episode are often more prolonged and constant throughout the day, include physical symptoms, whereas mood symptoms solely due to BPD are often triggered by external events and fluctuate throughout the day. Furthermore, the impulsive, aggressive, and interpersonal problems experienced in BPD are often not experienced in MDD (APA, 2013). Affective instability and impulsivity are commonly seen in both $\mathrm{BPD}$ and bipolar disorder patients. However, the mania that is experienced in Bipolar Disorder I is prolonged and constant compared to the affective instability experienced in BPD, which has a more fluctuating course that is contingent upon environmental cues (APA, 2013).

Persons with BPD have often experienced traumatic events and may experience symptoms of full or partial PTSD symptoms. While PTSD is common in persons with BPD, not all persons with BPD have PTSD, nor is PTSD necessary for a diagnosis of BPD (Zanarini et al., 1998). While the symptoms of negative cognitions and emotions such as dysphoria, shame, and guilt common in PTSD are also seen in BPD, the intrusion, hyper-vigilance, and avoidance symptoms necessary for a PTSD diagnosis are not typical of BPD. In addition, PTSD symptoms flare up due to cues or triggers in the environment, whereas symptoms of BPD are often rooted in interpersonal stressors such as rejection and criticism. Lastly, identity disturbance and the frantic efforts to avoid abandonment resulting in relational difficulties are not common in persons with PTSD (APA, 2013).

Persons with BPD and attention deficit hyperactivity disorder (ADHD) often demonstrate problems in attention and impulsivity or hyperactivity. However, persons with ADHD lack the identity disturbances, chronic suicidality, and sensitivity to abandonment seen in BPD. Persons 
with ADHD e also show symptoms of attention and hyperactivity issues early in the course of one's life (APA, 2013). Lastly, substance use disorders are commonly comorbid with BPD. Impulsivity, emotional lability, aggressiveness, and interpersonal problems are common across both conditions. However, many of the symptoms of substance use disorder emerge only while a person is taking substances and are often absent during remission, whereas the symptoms of BPD are persistent despite remission of substance use disorder (APA, 2013).

\section{OVERVIEW OF DIALECTICAL BEHAVIOR THERAPY}

DBT, developed by Marsha Linehan about 30 years ago, is a multifaceted, systemic model of therapy that utilizes components of cognitive behavioral therapy, behaviorism, and mindfulness (Linehan, 1987). The goal of DBT is to address emotional distress as well as the oftendestructive behaviors that result from BPD. In order to address both components, DBT therapists rely on a manualized framework that incorporates skills training, collaborative problem- solving, contingency management, and mindfulness that is based in a holistic philosophy (Linehan, 1987; O'Connel, 2014). The theoretical foundation of DBT is rooted in biosocial and developmental theories to understand the complex needs of individuals who are diagnosed with BPD (Koons, 2008). In recent years, DBT has also been adapted to treat substance use disorders, eating disorders with both adult and adolescent populations, as well as PTSD with various veteran populations.

\section{Historical Development}

DBT was developed by Marsha Linehan and her colleagues at the University of Washington, Seattle, in the late 1980s and early 1990s as a response to her clinical work with chronically suicidal women who were not responding to the standard treatments of the time (Koons, 2008). Most of Linehan's patients met the criteria for BPD, due to the impulsive behavior, mood instability, and the relational distress they exhibited (Koons, 2008). These characteristics led to prematurely leaving treatment and frequent emergency hospitalizations.

Linehan theorized that the common factor that her clients experienced involved an inability to effectively regulate emotions. Linehan believed that this inability to self-regulate led clients to avoid emotions in self-destructive ways such as through substance use and self-injurious behaviors. Linehan identified this emotional dysregulation as the core of the problematic and self-destructive behaviors that are common with individuals diagnosed with BPD. Linehan argued that emotional dysregulation contributed to relational distress, impulsive and high-risk behaviors, as well as anger outbursts and cognitive impairments such as paranoia, dissociation, and in some cases, hallucinations (Koons, 2008; Linehan, 1987). DBT was developed to address the specific needs of a population that had previously not responded well to traditional treatments. Linehan's model of treatment is intended to link emotional dysregulation with specific behavioral interventions that address the unique needs of individuals diagnosed with BPD (Crowell et al., 2009). Early studies conducted by Linehan and her colleagues found that over the course of a year, clients enrolled in the DBT program engaged in less parasuicidal behaviors, continued attendance in therapy, and experienced fewer hospital admissions than the control group that received treatment as usual (TAU; O'Connell \& Dowling, 2014). The 6- and 12-month follow-up evaluations reported a continued reduction in symptomatic behaviors in the DBT group compared to the TAU group (O'Connell \& Dowling, 2014).

\section{Central Theoretical Constructs}

DBT is rooted in biosocial theory that explains the etiology of BPD through the lens of biological vulnerabilities exacerbated by an invalidating social environment (Koons, 2008; Linehan, 1987). 
The symptoms of BPD have genetic, neurobiological, psychological (e.g., stable personality traits), and social (e.g., childhood maltreatment, trauma, early and sustained toxic stress) pathways (Gunderson et al., 2018; Skodol et al., 2002). The bio-social model of BPD positions BPD as an interaction between adverse life events such as trauma and child maltreatment, and genetic vulnerability to traits that are important to the disorder that can then lead to psychosocial problems in school, work, independent living, and personal relationships (Crowell et al., 2009; Distel et al., 2011; Gunderson et al., 2018; Linehan, 1987, 2015). Within this framework, BPD can be better considered as a combination of a disorder with a cluster of symptomatic experiences (e.g., mood and anxiety disturbance) and stable personality traits such as high antagonism, negative affectivity, and impulsivity (McGlashan et al., 2005; Skodol et al., 2005a).

Research has identified multiple neurobiological contributors to BPD. First, there is good evidence for the heritability of BPD, particularly for the personality traits of low agreeability and impulsivity (Kendler et al., 2008; Skodol et al., 2002; Torgersen et al., 2000). There is also some evidence suggesting overlap between the genes associated with impulsivity and affective instability in other major mental illness such as schizophrenia, bipolar disorder, MDD, and BPD (Witt et al., 2017). Abnormalities in the functioning and connectivity between the limbic system and prefrontal cortex may also lead to symptoms and functional impairments in learning, cognitive flexibility, memory, attention, planning, decision-making, problem-solving, impulsivity, anxiety, stress response, and emotional regulation commonly seen in persons with BPD (Ruocco, 2005).

Invalidating environments marked by the experience of childhood maltreatment in the form of physical and emotional abuse and neglect have been found to be associated with BPD (Widom et al., 2009). Linehan describes an invalidating social environment, primarily the family, in three ways: (1) an environment marked by negative, indifferent or demeaning interactions and that lacks health models of emotional expression; (2) an environment that is marked by interactions that are highly charged and emotionally toxic; and (3) a mismatch in temperament and parenting styles between a child and caregivers (Linehan, 2015). Linehan identifies three types of families that increase the probability of a child developing BPD. She describes (1) the disorganized family, which is characterized as neglectful or abusive; (2) the perfect family, which is characterized as avoidant of negative emotions; and (3) the normal family, in which there is a mismatch of temperament between caregivers and the child (Crowell et al., 2009; Linehan, 2015). These invalidating transactional social environments, combined with a child's biological vulnerabilities, can negatively impact a child's ability to emotionally regulate and manage behavior within a social context. Furthermore, the coping strategies developed during early emotional development are often maladaptive and become problematic in interpersonal and social functioning (Crowell et al., 2009; Koons, 2008; Linehan, 1987, 2015).

It should be noted that childhood sexual abuse, while often identified as an important contributor to BPD, has not been associated with an increased risk for BPD compared to physical abuse and neglect (Widom et al., 2009). Most people who are sexually abused do not develop BPD and many of those with BPD often have not experienced sexual abuse. However, while childhood abuse and neglect are correlated with BPD diagnoses, twin studies have found no causal link between abuse and later development of BPD traits. This research attributed the development of BPD to be more influenced by genetic vulnerabilities to pathological, emotional, and behavioral symptomologies (Bornovalova et al., 2013).

\section{Foundations of Dialectical Behavior Therapy}

DBT is a structured intervention that is based on three foundational theories: behaviorism, Zen, and dialectics. The combination of these theories addresses the consequences of the biological vulnerabilities and invalidating social environments identified as maladaptive in the Biosocial Theory. First, behaviorism emphasizes the importance of assessment, data collection, and analysis of the relationship between behavior and consequences. DBT utilizes similar interventions 
(e.g., problem-solving, skills training, contingency management, behavioral analysis, exposure, conditioning, and cognitive modification) to traditional cognitive behavioral treatment (CBT) and behavioral therapies. These interventions seek to understand how maladaptive behaviors are developed and how to replace and reinforce new, more effective behaviors (Koons, 2008; Linehan, 2015).

Second, Zen is an eastern philosophy based on the belief that knowledge of self is inherent in everyone. DBT has adapted this philosophy and developed the idea of the "wise mind." The wise mind is a synthesis of emotion and cognition that leads to emotion regulation and appropriate behavioral responses. To function in a state of wise mind, DBT teaches core mindfulness skills that incorporate "what skills," which are achieved through observation, description, and participation, and the "how skills." "How skills" include the acceptance of a non-judgmental stance, maintaining one-mindedness, and effectiveness (Koons, 2008; Linehan, 2015). The "what skills" allow the participant to notice and describe a situation from a non-emotional, here and now, factual place rather than from a place of emotional reactivity. The "how skills" allow the participant to accept a situation without judgment, remain focused on the here and now, as well as develop a response to the situation that effectively addresses the issue presented. This decreases emotional dysregulation and impulsivity and leads to thoughtful engagement and problem-solving. These mindfulness skills are central to DBT and are the primary way in which DBT differs from other behavioral therapies.

Third, the final tenet of DBT includes the western philosophical tradition of dialectics that espouses that truth is a synthesis of opposites. Dialectics understands that there is constant struggle among multiple factors and that change in one factor will cause a disruption of homeostasis and in other factors. A dialectical worldview directly challenges the dichotomous, "black and white" thinking that is common in individuals who are diagnosed with BPD. Dialectics encourages the viewpoint of "both/and" rather than an "either/or" perspective. This perspective allows for further exploration of alternative explanations and strategies for problem-solving. This is achieved when the client and the therapist can balance the concepts of acceptance and change. Acceptance is demonstrated through validation and empathy strategies, while change occurs through the implementation of behavioral interventions and skills training (Koons, 2008; Linehan, 2015).

\section{Structure of Treatment}

DBT is a highly structured treatment protocol that has several components. Each branch of the intervention provides support to the client and the therapist, and guides the course of treatment. DBT is most often delivered in outpatient settings due to the length of the treatment process. However, adaptations of DBT have been effectively deployed in hospital settings with good outcomes indicating that further study of DBT practices in inpatient settings is warranted (Bloom et al., 2012).

The first component is individual therapy. The role of the individual therapist is to manage and direct the treatment process. The therapist serves at the liaison to the treatment team, facilitates the development of treatment goals, and monitors progress. The therapist and the client meet once a week for one 50-minute session. During this meeting, the therapist addresses the established treatment hierarchy that has been collaboratively developed by the therapist and the client. The therapist also reinforces new behavioral skills and works to develop and maintain an effective and productive relationship with the client (Koons, 2008; Linehan, 1987, 2015).

The second component of DBT is skills training. This is the heart of DBT as is delivered in a group setting with other participants and two co-facilitators. The class is a two-hour session offered once a week and follows a set curricula that is broken into four modules. These modules build upon each other and always follow a set schedule of presentation. Participants are required to attend each of the modules and to successfully complete mandatory homework assignments outside of sessions. The modules are: (1) Mindfulness, (2) Distress Tolerance, (3) 
Emotion Regulation, and (4) Interpersonal Effectiveness. The completion of all four modules takes approximately 10 weeks. The cycles are then repeated with the same participants two more times in order for the participants to re-learn the skills multiple times. The entire process takes approximately a year. During this time, the client also participates in weekly individual therapy sessions with the primary therapist as described in the first component (Koons, 2008; Linehan, 1987, 2015).

The third component is therapist consultation sessions. Due to the stressful nature of working with high acuity clients, DBT therapists and skills group facilitators meet weekly to provide support and consultation about the treatment process and the needs of participants. During this consultation time, therapists are encouraged to hold each other accountable for maintaining fidelity to the model as well as problem solve specific issues that may be occurring during treatment with an individual client or with a cohort of participants in the skills training classes. This consultation also allows for direct communication between the primary therapist and the skills facilitators in order to reduce triangulation among the team members as well as ensure that all members of the team are addressing the same treatment goals (Koons, 2008; Linehan, 1987, 2015).

The fourth component, telephone coaching, is an integral part of the treatment process because it allows for clients to have access to their primary therapist in the time between sessions when emotional dysregulation can lead to self-destructive behavior. Clients are informed that this is not a therapy session, rather a brief intervention to review skills and monitor safety. Clients are informed that they are to contact the therapist prior to engaging in self-injurious, suicidal, or self-destructive behavior. If they self-injure or participate in any problematic behavior, they are prohibited from contacting the therapist for 24 hours. This is to ensure that the therapist is not reinforcing negative behavior (Koons, 2008; Linehan, 1987, 2015).

\section{Treatment Hierarchy}

In DBT, the primary therapist and the client collaborate to develop an individualized, structured treatment plan based on the stage of the disorder and the identification of problematic behaviors. Stage 1 of treatment is designed to address the most pervasive, impulsive, and dangerous behaviors. The treatment plan of stage 1 is divided into four target areas. The first target area is the behaviors that threaten life and safety. This includes homicidal or suicidal thoughts and actions, NSSI behaviors, and any other behaviors that could lead to death. The second target area is the behaviors that interfere with therapy. This includes noncompliance with treatment recommendations, nonattendance to sessions or the skills training, as well as refusal to complete assignments. The third target area focuses on the behaviors that negatively impact quality of life, such as substance abuse or disordered eating patterns. Finally, the fourth target area addresses behavioral replacement skills in which the client identifies and substitutes maladaptive coping behaviors with healthy, adaptive coping skills (Linehan, 2015).

Stage 2 of the treatment protocol occurs when there is a significant reduction in emotional and behavioral dyscontrol. While clients may continue to struggle with some emotion regulation, they are markedly less reactive and engage in less impulsive behavior. In stage 2 , the primary area of focus is on acceptance. This includes acceptance of trauma, its impact on the client's life and, most importantly, of self. Therapists must not move forward with stage 2 until the client has successfully learned and deployed skills to manage the effects of trauma and emotional and behavioral dyscontrol. Otherwise, the client may be more likely to return to negative behaviors and emotion dysregulation (Linehan, 2015).

Stage 3 targets "problems in living". Once the client reaches this stage of treatment, the focus shifts from emotional and behavioral regulation to increasing skills of living. This includes managing interpersonal conflicts, applying long-term problem-solving strategies, and managing multiple stressors that are common in everyday life. This stage utilizes several strategies that are common in CBT and other behavioral treatments (Linehan, 2015). 
Finally, stage 4 focuses on building a joyful and fulfilled life. This may include seeking out new relationships, engaging in new pursuits or hobbies, or achieving lifetime goals. The development of meaningful and purposeful activities and the establishment of health social networks are the main goals of this stage. Once these elements have been successfully achieved, the client may have a renewed sense of self, as well as confidence in their abilities to problem solve and live healthy, meaningful lives. At this point, the therapist and the client can begin the therapy termination process (Linehan, 2015).

\section{Core Strategies of Dialectical Behavior Therapy}

The core strategies of DBT are based in the dialectical perspective of acceptance and change. This is achieved through a balance of problem-solving skills and validation. The problem-solving skills utilized in DBT are similar to those in CBT and other cognitive and behavioral interventions. To accurately develop a treatment hierarchy, the therapist conducts a behavioral assessment. From this assessment, a problem is identified as well as the likely cause(s) and barriers to resolution of the problem. Upon completion of the assessment, the specific target behaviors are identified and a strategy for solving the problem is implemented through skills training, homework, and other behavioral interventions. Contingency management, shaping, extinction and punishment, exposure-based procedures, cognitive restructuring, and consequence tracking are commonly used behavioral and cognitive strategies (Koons, 2008; Linehan, 1987, 2015).

Validation strategies are an integral part of DBT and what separates it from traditional cognitive and behavioral interventions. Validation strategies include reciprocal communication, cheerleading, and general, emotional, behavioral, cognitive, and functional validation. The focus of the validation strategies is to soothe the client, diminish self-invalidation, teach emotion observation and labeling skills, and provide opportunities for open emotional expression. The purpose of validation strategies is to enable better self-thinking and behavior management abilities and to diminish negative self-assessment. Validation strategies are utilized in both individual and group sessions. The therapist or facilitator uses purposeful validation to increase the therapeutic alliance. Effective use of validation allows a client to feel heard and acknowledged by the therapist and facilitator, which creates opportunities for problem-solving. Oftentimes, validation is a useful strategy for de-escalation of emotional reactivity, which will allow for participation in situational problem-solving (Koons, 2008; Linehan, 1987, 2015).

\section{Empirical Support and Critique of the Dialectical Behavior Therapy Model}

DBT has been found to be one of the more effective psychotherapeutic approaches to treating BPD, particularly in reducing suicidal and parasuicidal behaviors, hospitalizations, and use of emergency room services, angry outbursts, and other behaviors linked to treatment dropout (Cristea et al., 2017; Linehan et al., 2006; Stoffers et al., 2012). DBT has been adapted over the years for use with different diagnoses and populations, including older adults, substance use disorders, eating disorders, and mood disorders. The results of these adaptations have demonstrated efficacy in the reduction of problem behavior, such as binge eating or substance use (Linehan \& Dexter-Mazza, 2014). DBT has also been modified to a skills-only group. This adaptation was found to decrease symptoms of mood disorders, emotional dysregulation, and symptoms of PTSD (Linehan \& Dexter-Mazza, 2014).

The primary critique of DBT is the difficulty of maintaining fidelity to the model in frontline clinical work. The model requires a considerable amount of staff and resources that are not always available to agencies. Furthermore, the amount of time and training required to practice DBT is a barrier to effective implementation of the model. The challenging nature of the population combined with the intensive requirements of the model can be stressful for clinicians and supervisors. Finally, empirical studies have also identified several other approaches to treating 
BPD with comparable outcomes to DBT indicating the need for further clinical trials that compare DBT with these other approaches in order to assess efficacy (Cristea et al., 2014; McMain et al., 2009).

\section{CASE STUDY 10.1}

Background information. Carrie is a 35-year-old Caucasian female referred by court order for outpatient assessment and treatment for mental illness and substance abuse. Carrie is a bright, engaging, and humorous individual. When asked what goals she would like to achieve as part of therapy, she states, "I just want to get my life somewhat stable so I can work a little and keep an apartment and not kill myself or anyone else." In the past year, she has lost two jobs and has been forced to move twice due to her aggressive behavior and substance use. For 6 months, she was homeless "surfing on my friends' couches." Currently, she has a studio apartment on the south side of the city. She is not currently employed and is living off some savings, support from parents and her small unemployment check from her last job, which will run out in two months.

During the initial intake assessment, Carrie reveals that she recently relapsed after two years of sobriety from cocaine. She began abusing substances again when she lost her job as a store clerk. When asked about the circumstances of her termination, she states, "Those people were just looking for a reason to get rid of me. They were always talking about all the things I did wrong. I got mad and told them off." After her dismissal, she tells you that she went on a cocaine and alcohol binge for several days. During this binge, she was arrested for assault. It is this incident that precipitated her referral to treatment.

When probed about the circumstances of the assault. Carrie states, "I was at a bar and I was drinking. I was in the bathroom, watching this woman next to me and she was so beautiful. She had gorgeous hair. I asked her if I could borrow her brush and she looked at me funny. She said, 'no,' and started to walk out. She pissed me off. She looked at me like I was a piece of crap. So, I grabbed her by her hair, spun her around and punched her in the face. Then all the security came in and arrested me. I think the band at the bar set me up to meet her in the bathroom because they didn't want me there."

During the assessment, Carrie discloses a childhood history of sexual and physical abuse from a neighbor and an uncle. She has also been in a number of physically and sexually abusive adult relationships. She reports that she briefly became a sex worker at the age of 25 to support her cocaine use. Carrie has numerous arrests, but this is her first possible felony charge. She reports being arrested for getting into fights, stalking a former boyfriend, and disorderly conduct. Most of these incidents occurred within the context of a relationship with a man. She has never been married and has a history of multiple relationships, many of which involve interpersonal violence. You probe further about her relationships and she reveals that her last two relationships ended because of her anger. She states, "They would say things to me or not want to come over and I would get mad. I'd curse them out and then when I wasn't mad anymore I would call them all the time. They would just stop answering the phone or they would put a restraining order on me because I would stalk them at home and at work ... you know, sometimes this is just too hard. I get so lonely and when I am not in a relationship I often don't really know who I am. My former therapist said that I need to be in a relationship because I can't stand to be with myself and I think she was right. If I didn't think I would go to hell, I would probably kill myself."

During these episodes, Carrie reported that she often cuts herself with a razor or burns herself with lit cigarettes. She will also call her friends or partners and threaten to kill herself if they do not answer her calls. She has had one serious suicide attempt in which she took a handful of benzodiazepines and drank a significant amount of alcohol. She needed to have her stomach pumped at the ED and was in the hospital for several days. She denies that it was an attempt at 
suicide, but rather, "a mistake." She states, "If I really wanted to die I wouldn't have called my friend right after I took the pills. I was just so sad. It got blown out of proportion and I ended up in the hospital for a week."

\section{Diagnosis}

In order to determine if Carrie is an appropriate candidate for DBT, the therapist must conduct a diagnostic and pretreatment assessment. Based on the information provided, it is likely that Carrie meets the criteria for BPD. Most importantly, she exhibits a long pattern of (1) suicidal behavior and self-injury, (2) emotional dysregulation, (3) frantic efforts to avoid abandonment, (4) identity disturbance, (5) chronic feelings of emptiness, (6) substance use and other impulsive and reckless behaviors, (7) chronic anger and aggression, and (8) unstable and often violent relationships. This pattern of behavior has caused significant distress and impairment resulting in the loss of relationships, employment, and stable housing.

\section{Treatment Approach}

The following section illustrates the various stage-based treatment goals for Carrie as outlined in Linehan's DBT approach (Linehan, 2015). At the beginning of the treatment relationship, the therapist will assess for Carrie's appropriateness for DBT. Carrie must be agreeable to participating in all aspects of DBT as well as agreeing to address the behaviors that will be targeted. The therapist will provide psychoeducation regarding the Biosocial Theory of BPD and orient Carrie to the expectations of DBT. This will include a discussion of the interventions that will be utilized, including the coaching calls and skills training. Once the rules of DBT have been established and Carrie has consented to participate, Carrie and the therapist will enter into a therapeutic relationship. The therapist and Carrie will conduct a comprehensive analysis of the target behaviors and determine the appropriate level of care and interventions.

In stage 1 of DBT, the following behaviors will be targeted: (1) life-threatening behaviors (suicidal behavior, threats of suicide, self-harming [cutting] behavior), (2) therapy-interfering behaviors (history of noncompliance with treatment providers), and (3) quality-of-life-interfering behaviors (homelessness, unemployment, substance abuse, intimate partner violence, and behavior dyscontrol with serious consequences).

Upon the successful management of stage 1 behaviors, the underlying mental health issues and trauma can begin to be addressed in stage 2. For Carrie, this means that she has demonstrated the ability to utilize adaptive problem-solving and situational coping skills, resolved her employment and housing situation, and no longer engages in substance use or self-harming behaviors. In stage 2 the therapist can move toward (1) addressing her trauma symptoms through prolonged exposure and developing grounding and other coping skills to manage trauma symptoms as they arise and (2) developing strategies to cope with severe emotional dysregulation. The focus of stage 2 will also be to help Carrie develop acceptance of her trauma and her emotional dysregulation symptoms, and to begin to develop a sense of self and selfworth. In stage 3, the therapist will focus on helping Carrie develop and pursue goals, manage interpersonal distress, and develop coping skills as they apply to the development and maintenance of healthy interpersonal relationships with intimate partners, friends, family, and coworkers. Lastly, stage 4 will focus on helping Carrie build a joyful and fulfilled life through developing healthy and meaningful pursuits.

Based on the targeted behaviors, the recommended treatment includes individual treatment, which would include a focus on tracking and challenging behaviors using diary cards and behavior chain analysis of those behaviors. It will also be necessary for Carrie to be involved in the group skills training groups. Due to the severity of Carrie's impulsive behaviors, it is likely that Carrie will need regularly scheduled coaching calls in between individual sessions and skills training sessions. Due to the number of psychosocial stressors Carrie is experiencing, it 
may be beneficial to also include case management services that are DBT informed. It is also important to note that during treatment, the therapist who is working with Carrie must be actively involved with the other members of the DBT consultation team. Insurance companies and agency capacity often determine treatment length. Most programs require 6 to 12 months of treatment based upon the severity of the issues presented by the client.

\section{SUMMARY POINTS}

In summary, this chapter has:

- described the prevalence, pathogenesis, symptomology, and course for BPD;

- explained the biological, psychological, and social factors that contribute to the development of BPD;

- described screening and assessment practices for the effective diagnosis of BPD;

- explored the historical development, central theoretical constructs, structures, processes, evidence base, and limitations of DBT; and

- described how to identify and effectively apply the specific practices and interventions of DBT.

\section{KEY REFERENCES}

Only key references appear in the print edition. The full reference list appears in the digital product found on http:/ / connect.springerpub.com/content/book/978-0-8261-6556-5/part/sec03/part/sec032/ chapter/ch10

American Psychiatric Association. (2013). Diagnostic and statistical manual of mental disorders (DSM5) (5th ed.). American Psychiatric Association.

American Psychiatric Association. (2001). Practice guideline for the treatment of patients with borderline personality disorder. American Journal of Psychiatry, 158, 1-52.

Black, D. W., Blum, N., \& Hale, N. (2004). Suicidal behavior in borderline personality disorder: Prevalence, risk factors, prediction and prevention. Journal of Personality Disorder, 18(3), 226-239.

Bloom, J. M., Woodward, E. N., Susmaras, T., \& Pantalone, D. W. (2012). Use of dialectical behavior therapy in inpatient treatment of borderline personality disorder: A systematic review. Psychiatric Services, 63(9), 881-888.

Bornovalova, M. A., Huibregtse, B. M., Hicks, B. M., Keyes, M., McGue, M., \& Iacono, W. (2013). Tests of a direct effect of childhood abuse on adult borderline personality disorder traits: A longitudinal discordant twin design. Journal of Abnormal Psychology, 122(1), 180-194.

Cristea, I. A., Gentili, C., Cotet, C. D., Palomba, D., Barbui, C., \& Cuijpers, P. (2017). Efficacy of psychotherapies for borderline personality disorder: A systematic review and meta-analysis. JAMA Psychiatry, 74, 319-328. https://doi.org/10.1001/jamapsychiatry.2016.4287

Crowell, S. E., Beauchaine, T. P., \& Linehan, M. M. (2009). A biosocial developmental model of borderline personality disorder: Elaborating and extending Linehan's theory. Psychological Bulletin, 135(3), 495-510.

Distel, M. A., Middeldorp, C. M., Trull, T. J., Derom, C. A., Willemsen, G., \& Boomsma, D. I. (2011). Life events and borderline personality features: The influence of gene-environment interaction and gene-environment correlation. Psychological Medicine, 41, 849-860. https:/ /doi.org/10.1017/ S0033291710001297

Donegan, N. H., Sanislow, C. A., Blumberg, H. P., Fulbright, R. K., Lacadie, C., Skudlarski, P., Gore, J. C., Olson, I. R., McGlashan, T. H., \& Wexler, B. E. (2003). Amygdala hyper-reactivity in borderline personality disorder: Implications for emotional dysregulation. Biological Psychiatry, 54, 1284-1293.

Grant, B. F., Chou, S. P., Goldstein, R. B., Huang, B., Stinson, F. S., Saha, T. D., Smith, S. M., Dawson, D. A., Pulay, A. J., Pickering, R. P., \& Ruan, W. J. (2008). Prevalence, correlates, disability, and comorbidity of DSM-IV borderline personality disorder: Results from the wave 2 national epidemiologic survey on alcohol and related conditions. Journal of Clinical Psychiatry, 69, 533-545. 